\title{
Lateral Transorbital Endoscopic Access to the Hippocampus, Amygdala, and Entorhinal Cortex: Initial Clinical Experience
}

\author{
H. Isaac Chen ${ }^{a} \quad$ Leif-Erik Bohman ${ }^{a} \quad$ Lyndsey Emery ${ }^{b} \quad$ Maria Martinez- \\ Lage $^{b} \quad$ Andrew G. Richardson ${ }^{a}$ e Kathryn A. Davis ${ }^{c}$ John R. Pollard ${ }^{c}$ \\ Brian Litt ${ }^{c, e}$ Roberta E. Gausas ${ }^{d}$ Timothy H. Lucas ${ }^{a, e}$ \\ Departments of a Neurosurgery, ${ }^{b}$ Pathology and Laboratory Medicine, ${ }^{c}$ Neurology and \\ ${ }^{\mathrm{d}}$ Ophthalmology, Perelman School of Medicine, University of Pennsylvania, and ${ }^{\mathrm{e}} \mathrm{Center}$ for \\ Neuroengineering and Therapeutics, University of Pennsylvania, Philadelphia, Pa., USA
}

\section{Key Words}

Amygdalohippocampectomy · Endoscopy · Epilepsy · Temporal lobectomy · Transorbital approach

\begin{abstract}
Background/Aims: Transorbital approaches traditionally have focused on skull base and cavernous sinus lesions medial to the globe. Lateral orbital approaches to the temporal lobe have not been widely explored despite several theoretical advantages compared to open craniotomy. Recently, we demonstrated the feasibility of the lateral transorbital technique in cadaveric specimens with endoscopic visualization. We describe our initial clinical experience with the endoscope-assisted lateral transorbital approach to lesions in the temporal lobe. Methods: Two patients with mesial temporal lobe pathology presenting with seizures underwent surgery. The use of a transpalpebral or Stallard-Wright eyebrow incision enabled access to the intraorbital compartment, and a lateral orbital wall 'keyhole' opening permitted visualization of the anterior temporal pole. Results: This approach afforded adequate access to the surgical target and surrounding structures and was well tolerated by the patients. To the best of our knowledge, this report constitutes the first case series describing the endoscope-assisted lateral transorbital approach to the temporal lobe. We discuss the limits of exposure, the nuances of opening and closing, and comparisons to open craniotomy. Conclusion: Further prospective investigation of this approach is warranted for comparison to traditional approaches to the mesial temporal lobe.


Chen et al.: Lateral Transorbital Endoscopic Access to the Hippocampus, Amygdala and Entorhinal Cortex: Initial Clinical Experience

\section{Introduction}

The past few decades have witnessed the development of a number of minimally invasive surgical approaches to the intracranial space. Pituitary surgery is the most evident case. Transsphenoidal surgery is considered the standard of care not only for routine pituitary tumors [1, 2] but also for suprasellar lesions like craniopharyngiomas [3]. Concurrent advances in neuroendoscopy and other surgical equipment have enabled the expansion of minimally invasive approaches through corridors other than the nasopharynx, including the posterior fossa [4] and the orbit. Transorbital approaches to the skull base and cavernous sinus spare patients the need for large craniotomies and brain retraction [5]. However, these pioneering techniques are not commonly used due to the relatively rare nature of the lesions that require an intracranial trajectory medial to the globe.

Surgical resection of the medial temporal structures is the definitive treatment for intractable temporal lobe epilepsy in the setting of mesial temporal sclerosis [6]. Recently, we demonstrated the feasibility of the lateral transorbital approach for access to the medial temporal lobe in cadaveric specimens, which we named transorbital endoscopic amygdalohippocampectomy (TEA) [7]. This approach permits a wide intracranial field of view with minimal displacement of the globe, allowing up to $97 \%$ of the hippocampus to be resected by volumetric measurements. Complete resection of the uncus and entorhinal cortex is also possible, which allows unrestricted visualization of the cerebral peduncle, cranial nerves, and arteries within the ambient cistern. Perhaps more importantly, the lateral cortex and white matter remain undisturbed.

Here, we present two cases of medial temporal lesions that underwent endoscopeassisted transorbital resection. These cases are compared to classical open approaches to the medial temporal lobe. We provide a critical appraisal of the relative merits and disadvantages of lateral transorbital endoscopic surgery.

\section{Patients and Methods}

Patients

Both patients presented for surgical evaluation of medial temporal lobe lesions after the onset of seizures. Salient features of their cases are summarized below.

Patient A is a 60-year-old, right-handed Korean male with a 5-year history of complex partial seizures. His seizure semiology was characterized by 20 -second staring spells occurring once a week. These seizures were poorly controlled with levetiracetam. On neurological examination, the patient was intact. His past medical history was notable for a left parietal 'glioma', which was diagnosed 10 years earlier by a biopsy performed at another institution. The histological slides were not available for our review. Magnetic resonance imaging (MRI) demonstrated an abnormal fluid-attenuated inversion recovery (FLAIR) signal in the left mesial temporal lobe and hippocampal sclerosis (fig. 1). The size of the FLAIR abnormality had increased since 2007, raising the suspicion for recurrent neoplasm.

Patient B is a 43-year-old, right-handed Caucasian female with a 1-year history of complex seizures consisting of staring spells, lip smacking, and finger rigidity with the recent onset of a generalized tonic-clonic seizure. On neurological examination, the patient was intact. MRI of the brain revealed an enlargement of the right amygdala and entorhinal cortex, raising the suspicion for neoplasm (fig. 2).

Procedures

Prior to surgery, both patients underwent comprehensive evaluations by a fellowship-trained epileptologist, neurosurgeon, and oculoplastics surgeon, which included a history, neurological examination, ophthalmological examination (i.e., visual field and acuity testing and fundoscopic examination), baseline laboratory tests, and thin-cut computed tomography (CT) and MRI studies for surgical planning. In both cases, the primary goal of surgery was to obtain a tissue diagnosis given the concern for neoplasm. Informed consent for a transorbital approach was obtained after a detailed discussion of the risks, benefits, and 


\begin{tabular}{l|l}
\hline ORL 2015;77:321-332 \\
\hline DOI: 10.1159/000438762 & $\begin{array}{l}\text { @ 2015 S. Karger AG, Basel } \\
\text { www.karger.com/orl }\end{array}$ \\
\hline
\end{tabular}

Chen et al.: Lateral Transorbital Endoscopic Access to the Hippocampus, Amygdala, and Entorhinal Cortex: Initial Clinical Experience
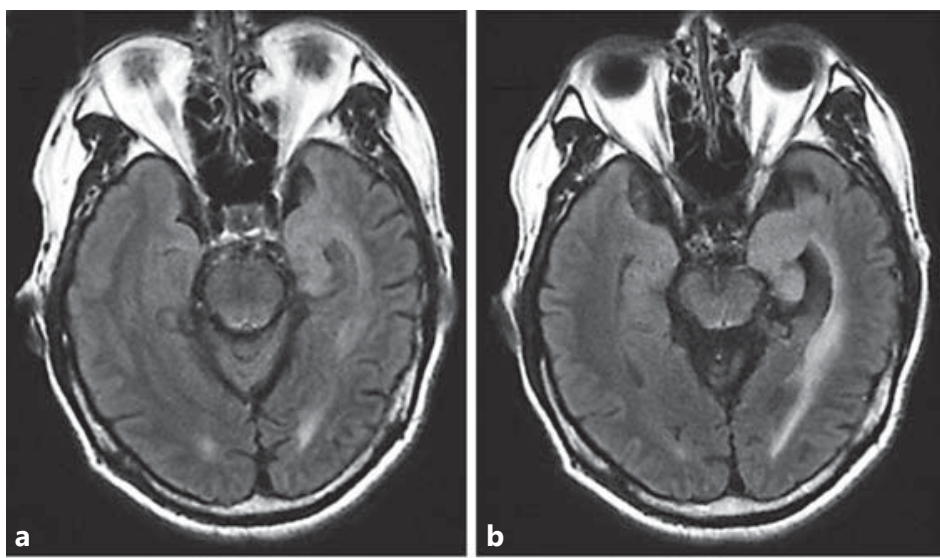

Fig. 1. Preoperative MRI for patient A. FLAIR MRI sequences demonstrate increased signal and expansion of the left hippocampus and mesial temporal structures [inferior (a), superior (d)]. White matter signal change in the temporo-occipital region reflects treatment change from the patient's prior chemoradiation.
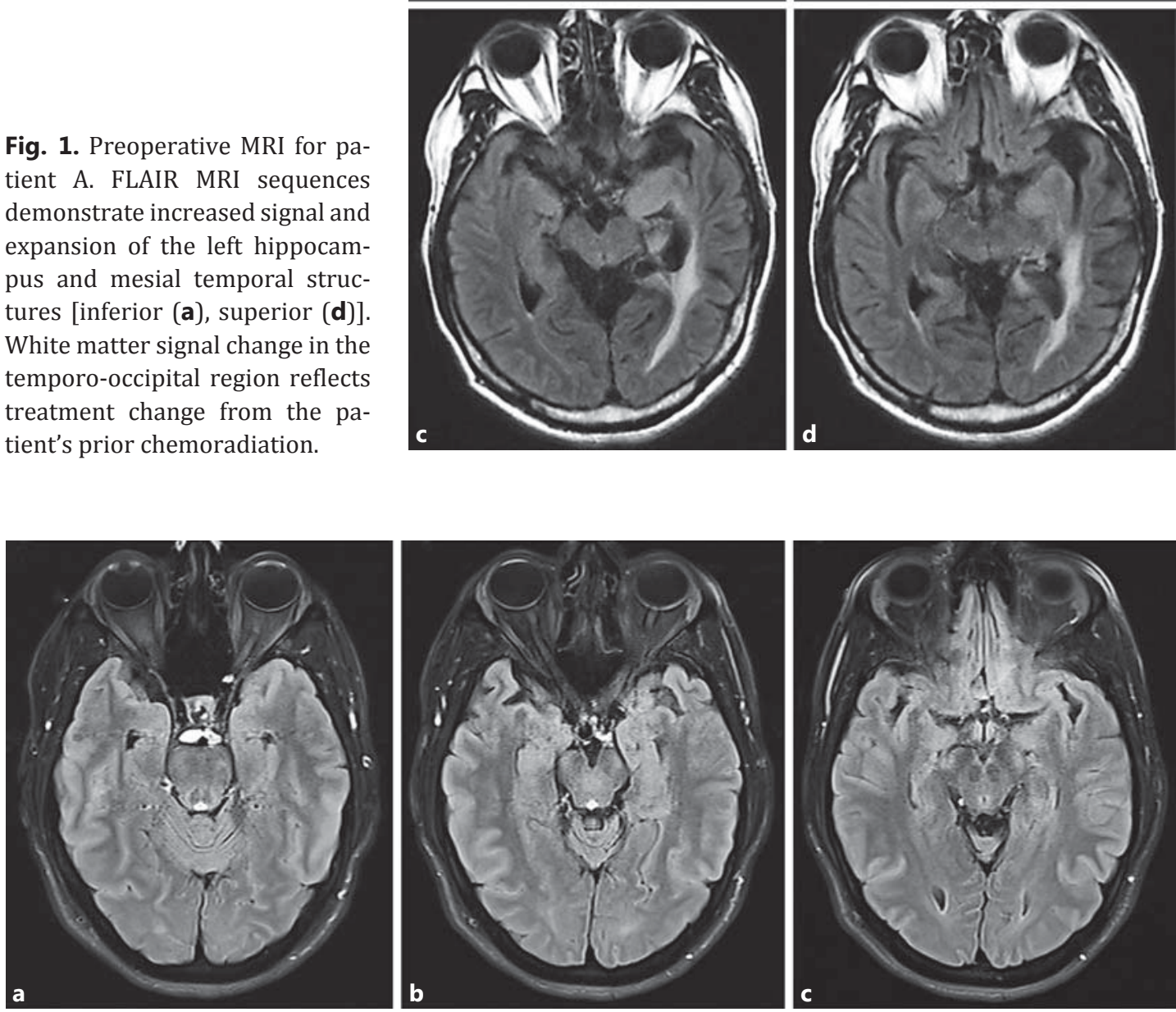

Fig. 2. Preoperative MRI for patient B. On these FLAIR MRI sequences, there is fullness of the right mesial temporal lobe structures, especially the amygdala [inferior (a), superior (c)].

treatment alternatives. This study was approved by the Institutional Review Board at the University of Pennsylvania.

Patients were positioned supine in a neutral orientation with the head stabilized in a Mayfield head holder. The neuronavigation system was registered using preoperative 3-tesla volumetric T1 sequences, which were fused to FLAIR sequences. The head and face were prepped with a dilute Betadine solution. Standard doses of antibiotics and dexamethasone were administered before incision. 
Chen et al:: Lateral Transorbital Endoscopic Access to the Hippocampus, Amygdala, and Entorhinal Cortex: Initial Clinical Experience

Fig. 3. Palpebral incision for patient B.

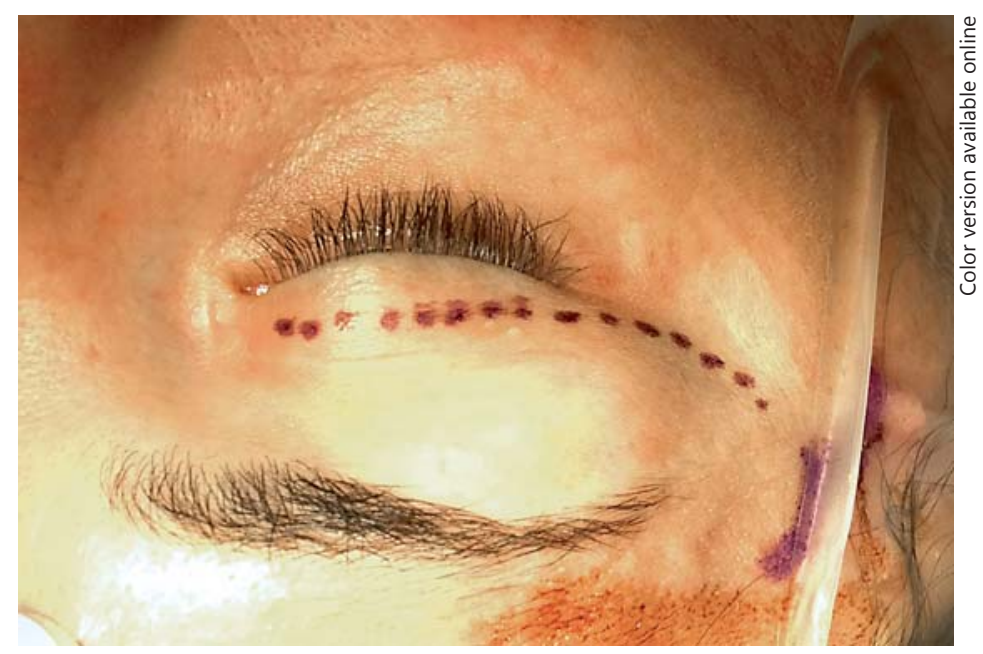

Prior to beginning the orbital dissection, a frontal ventriculostomy catheter was placed using standard anatomical landmarks to permit cerebrospinal fluid (CSF) drainage and intraventricular irrigation. In patient A, a lateral orbitotomy was initiated via a Stallard-Wright approach through a skin incision below the eyebrow that followed the curve of the lateral orbital rim extending laterally toward the temporal fossa. This incision was chosen because of the patient's eyelid anatomy. In patient B, a palpebral incision was performed (fig. 3). In both patients, the orbicularis oculi was divided in line with the muscle fascicles, and the dissection was carried down to the periosteum of the orbital rim. Along a subperiosteal plane, the periorbita was freed both along the internal wall of the orbit and externally into the temporal fossa, thereby further exposing the orbital rim. Subperiosteal blunt dissection within the orbit continued posteriorly along the lateral orbital wall until the inferior orbital fissure was identified (fig. 4a). The orbital soft tissue was gently retracted with a handheld malleable retractor to avoid injury to the globe, optic nerve, and extraocular muscles.

In patient $\mathrm{A}$, an additional orbital osteotomy of the lateral orbital wall from the frontozygomatic suture superiorly to the orbital floor inferiorly was performed to facilitate medial access and complete resection of the amygdala and hippocampus. For both patients, a keyhole orbitotomy between 1 and $1.5 \mathrm{~cm}$ in diameter was drilled in the greater wing of the sphenoid between the superior orbital fissure and the inferior orbital fissure (fig. 4b), and the bony edges were waxed. At this point, $20 \mathrm{ml}$ of CSF was drained from the ventriculostomy to facilitate brain relaxation. The dura was then incised in a U-shaped fashion, based medially. The anterior temporal pole could be identified immediately through the durotomy (fig. 4c). A trajectory to the head of the hippocampus was plotted using the neuronavigation system.

A 1-cm corticectomy was performed in the anterior temporal pole (fig. 4d). Deeper dissection to the temporal horn of the lateral ventricle was performed using endoscopic visualization (fig. 4e). A pneumatic endoscope holder (Mitaka USA, Inc.) permitted a bimanual dissection technique. Upon entering the ventricle, the pes of the hippocampus was visualized. In patient A, a hippocampal specimen was sent for frozen pathology, and then an amygdalohippocampectomy was performed using a combination of endoscopic dissectors, ring curettes, and suction. The hippocampal sulcus was identified during the dissection, which served as an anatomical landmark to guide hippocampal removal. In patient B, we used neuronavigation guidance to target the abnormal MRI signal within the entorhinal cortex and subjacent white matter. This tissue was rubbery in texture, and it was resected with ring curettes and sent for pathological analysis. In both cases, intermittent egress of CSF facilitated brain relaxation.

Once the desired brain resection was completed, hemostasis was achieved using bipolar diathermy and application of thrombin-soaked Gelfoam. The ventricular system was irrigated to clear debris by infusing saline through the ventricular drain. The dura was loosely reapproximated with a 6-0 absorbable suture. This dural closure was augmented with a free local tissue graft. Dural sealant was applied, and the orbitotomy defect was covered with Suprafoil, a tailored sheet of smooth nylon. The lateral orbital window in patient A was closed by reapproximating the lateral orbital wall with suture. The orbital periosteum, orbicularis oculi muscle, and skin were closed in layers, and a drain under bulb suction was placed in the temporal fossa adjacent to the lateral orbital wall. Steri-strips were applied to the incision. 
Fig. 4. Intraoperative photos from patient B. a Mobilization of the orbit medially reveals the frontozygomatic suture (arrowhead) running along the lateral orbital wall and the inferior orbital fissure (probe). b An orbitotomy is performed superior and lateral to the inferior orbital fissure. c The dura is opened in a Ushaped fashion based medially, which allows the temporal pole to be visualized. d Superficial dissection can be performed using the operating microscope. e Visualization of the deeper structures is facilitated using an endoscope.
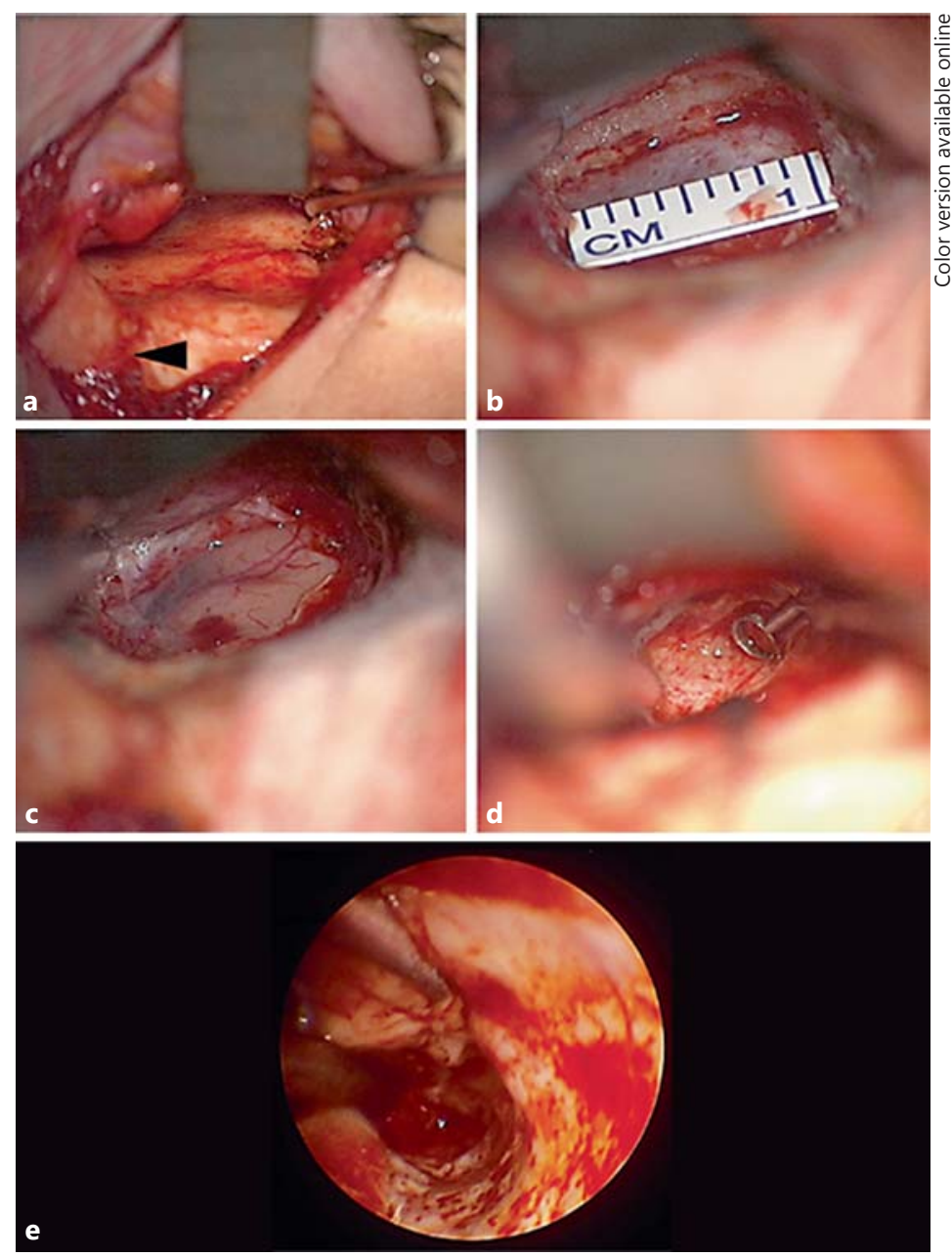

Including the time for placement of the ventriculostomy catheter, the operative time was 492 and 375 min for patients A and B, respectively. In comparison, the operative time for 5 recent anterior temporal lobectomies (ATL) performed by the senior author was $246 \pm 72$ min (mean \pm standard deviation), which was significantly less time (one-tailed t test of two samples with equal variance, $p=0.01$ ).

\section{Results}

\section{Pathology}

Pathological specimens from both patients were reviewed with frozen and permanent histological and immunohistochemical analysis. Patient A had evidence of hippocampal gliosis (see online suppl. fig. S1; see www.karger.com/doi/10.1159/000438762 for all online suppl. material), and patient B had evidence of entorhinal gliosis (online suppl. fig. S2). These pathological results verified that the desired surgical targets had been resected and did not disclose neoplastic processes in either case.

\section{Immediate Postoperative Course}

Both patients returned to their neurological and ophthalmological baseline immediately following surgery. Routine visual acuity and pupillary response assessments were performed 


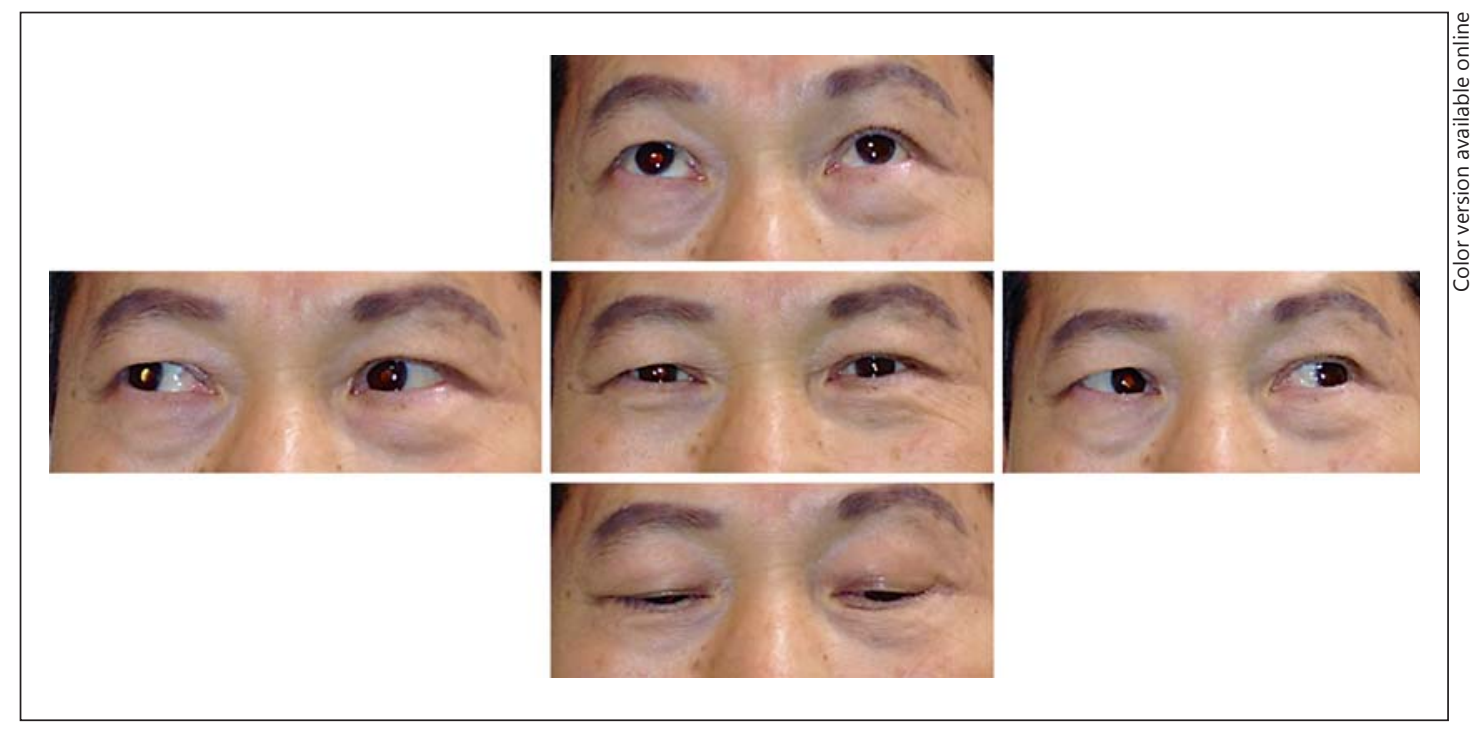

Fig. 5. Postoperative extraocular movements for patient A. At 4 months postoperatively, the patient demonstrates a full range of extraocular movement.

Fig. 6. Cosmetic result for patient B. At 6 months postoperatively, no evidence of cosmetic disfigurement is noticeable from the palpebral incision.

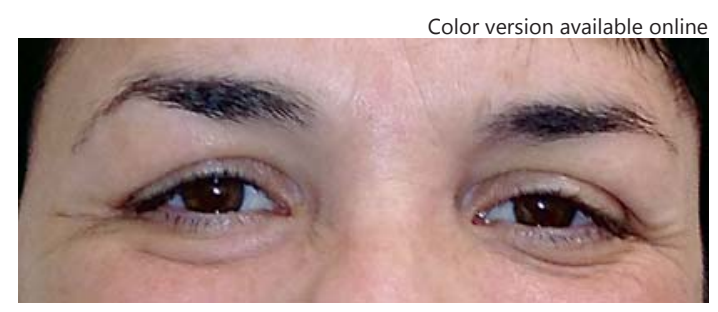

after surgery. The ventricular drains were weaned gradually. Both patients received routine postoperative imaging.

Patient B developed periorbital swelling when the ventricular drain was clamped, which was consistent with the diagnosis of an orbital pseudomeningocele. She denied any diplopia or changes in visual acuity. When the drain was opened, the symptoms resolved. The patient was offered surgical exploration or internalization of the ventricular drain. She opted for a ventriculoperitoneal shunt, after which the orbital pseudomeningocele did not recur.

Both patients were discharged with normal neurological and ophthalmological exams.

\section{Long-Term Outcomes}

We examined these patients in terms of clinical, cosmetic, and radiographic outcomes for 12 months after surgery. Clinical outcomes included the patients' seizure, neurological, and ophthalmological status.

Both patients have remained seizure free since surgery (i.e., Engel class I). With evidence of gliosis on the final pathology in both cases, no additional surgery was considered necessary. Formal ophthalmological testing 8 weeks after surgery documented baseline visual acuity, visual fields, and extraocular movements in both patients (fig. 5). Patient A experienced transient episodes of diplopia while watching television, which spontaneously abated about 


\begin{tabular}{l|l}
\hline ORL 2015;77:321-332 \\
\hline DOI: 10.1159/000438762 & $\begin{array}{l}\text { @ } 2015 \text { S. Karger AG, Basel } \\
\text { www.karger.com/orl }\end{array}$ \\
\hline
\end{tabular}

Chen et al.: Lateral Transorbital Endoscopic Access to the Hippocampus, Amygdala, and Entorhinal Cortex: Initial Clinical Experience

Fig. 7. Orbital craniectomy. Three-dimensional reconstruction of the pre- (a) and post- (b) dissection CT scans for patient B. This view of the skull demonstrates the orbital craniectomy superior to the inferior orbital fissure and lateral to the superior orbital fissure. Neither of these skull base foramina is violated.
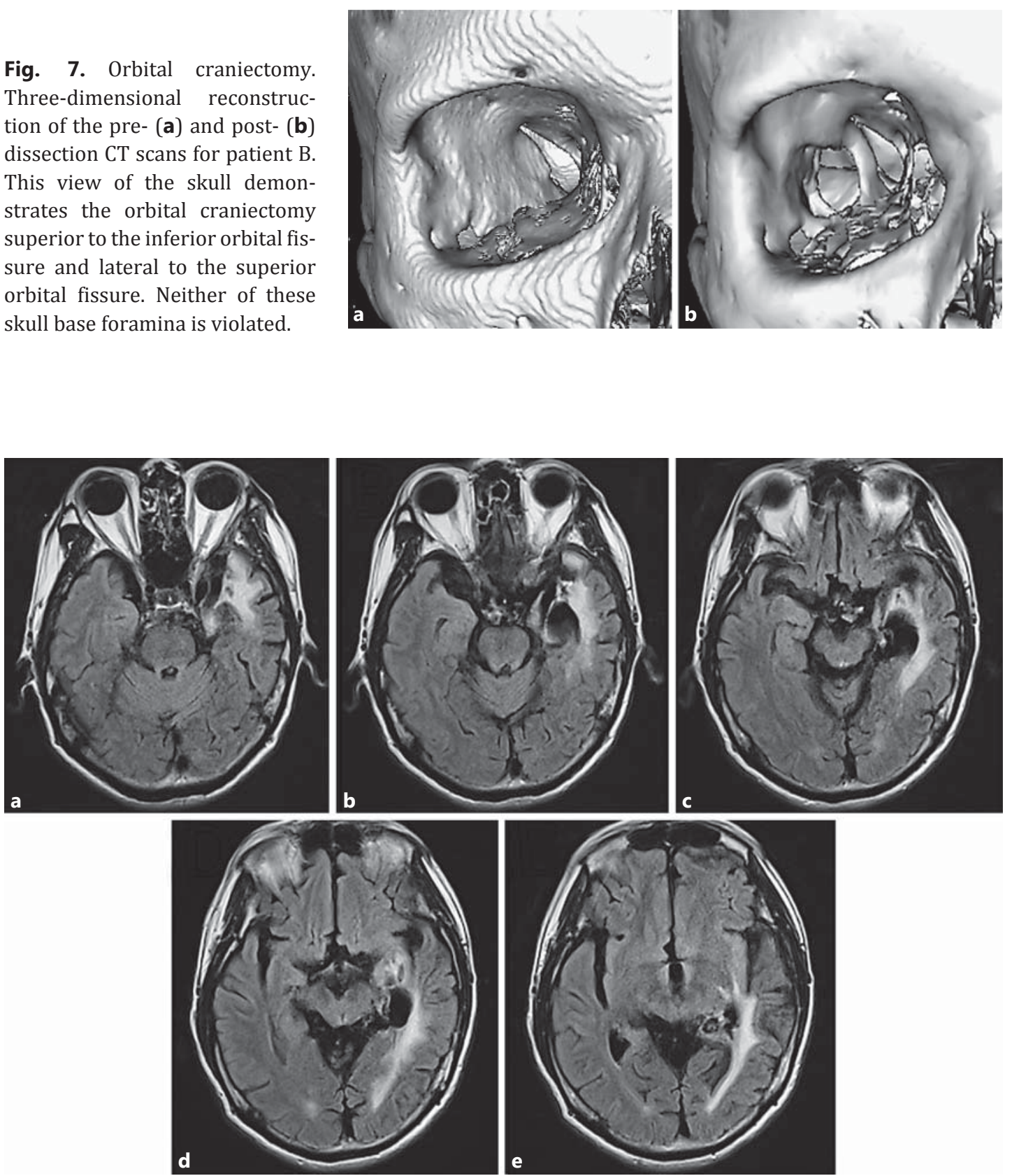

Fig. 8. Postoperative MRI for patient A. At 9 months postoperatively, a resection cavity back to the level of the quadrigeminal cisterns is seen on these FLAIR images [inferior (a), superior (e)]. The hippocampus and mesial temporal lobe have been completely removed.

3 months after surgery. At the 1-year mark, both patients were neurologically intact and had returned to their normal activities. Patient $\mathrm{B}$ had returned to running half marathons and had participated in a number of races. The cosmetic result in both cases was rated as 'excellent' by both patients and their families (fig. 5,6 ).

The extent of bone removal is illustrated with pre- and postoperative three-dimensional reconstructions of fine-cut CT scans (fig. 7). The extent of mesial temporal lobe resection is 
Fig. 9. Postoperative MRI for patient $\mathrm{B}$. A resection cavity encompassing the temporal pole and entorhinal cortex up to the amygdala is observed on these FLAIR images [inferior (a), superior (d)]. The surrounding brain is relatively normal.
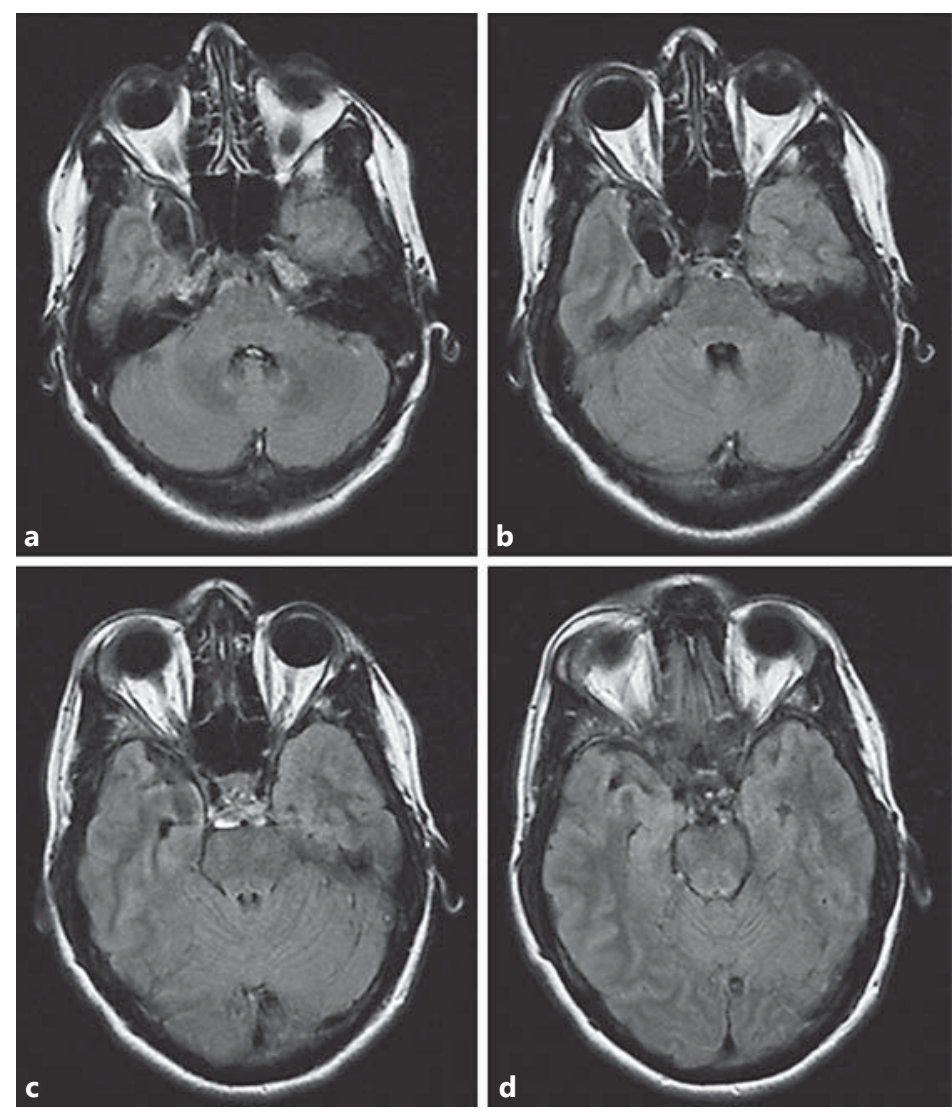

illustrated in the postoperative MR images. At least $95 \%$ of the amygdala and hippocampus was resected as determined by volumetric measurements in patient A (fig. 8), and a majority of the amygdalar complex abnormality as visualized on FLAIR sequences was removed in patient B (fig. 9).

\section{Discussion}

This case series is the first clinical description of a transorbital endoscopic approach to the medial temporal lobe via a lateral orbitotomy. The medial transorbital approach has been described in several clinical series for the repair of CSF leaks and skull base fractures, optic nerve decompression, and resection of extra-axial tumors [5, 8]. Previously, we [7] and others [9] have demonstrated the feasibility of a lateral transorbital approach to the intracranial space in cadaveric specimens. We have translated this approach into the clinical setting in the current series. To the best of our knowledge, this is the first example of using a transorbital approach to resect intra-axial brain lesions. It is possible that transorbital endoscopic surgery could be combined with other endoscopic techniques and robotic technology to broaden access to the intracranial space $[10,11]$.

With the lateral transorbital approach, surgical access to the amygdala, hippocampus, and entorhinal cortex was achieved successfully. Accurate localization of the targeted lesions was confirmed with intra-operative neuronavigation, pathology, and postoperative imaging. Both patients had normal neurological and ophthalmological examinations after surgery. Our 

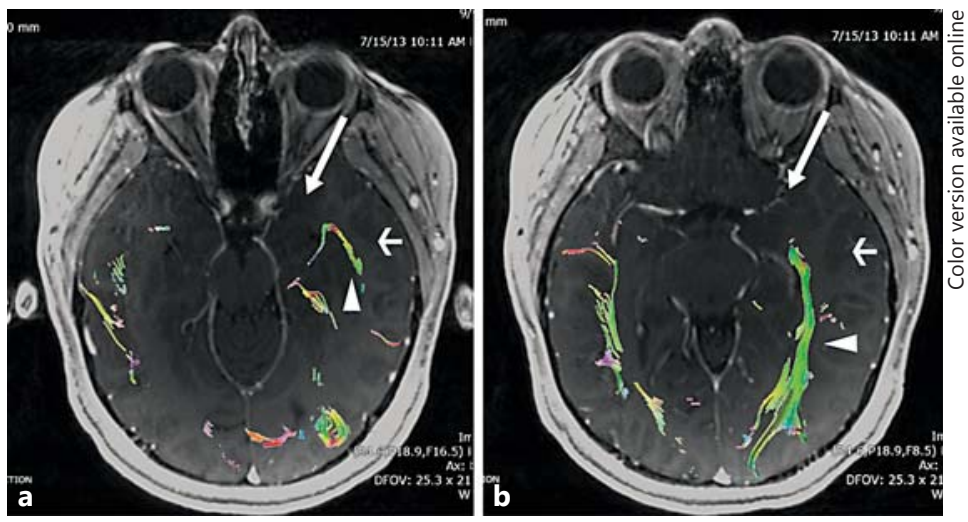

Fig. 10. Relationship of white matter tracts to surgical approaches. The optic radiations (a, arrowhead) and inferior longitudinal fasciculus/inferior fronto-occipital fasciculus (b, arrowhead) are at risk of injury from traditional approaches to the mesial temporal lobe (short arrows). TEA provides a direct route to the mesial temporal lobe through the temporal pole that avoids these white matter tracts (long arrows), which could translate into better neurological and cognitive outcomes.

results demonstrate that TEA is a feasible surgical approach to the medial temporal lobe for select indications.

Critically assessing the merit of TEA as an alternative to traditional approaches will require long-term clinical follow-up in a larger series of patients. There is also a need to compare TEA with other minimally invasive interventions for mesial temporal lobe pathology, such as endoscopic transventricular hippocampectomy [12], laser interstitial thermal therapy [13], and stereotactic radiosurgery $[14,15]$. The medial temporal lobe can be accessed using an occipital, transventricular route, but this approach has received little attention recently, in part because of the long working distances required. Laser interstitial thermal therapy involves the placement of a catheter in the area of interest and thermal ablation of tissue using laser energy. Each of these approaches has distinctive advantages and disadvantages that could predispose them for different indications. In the meantime, we may draw inferences from this initial clinical experience that inform our evaluation of this novel technique.

\section{Potential Benefits of TEA}

TEA provides a novel surgical approach to the mesial temporal lobe. To be appropriate for wider adoption, however, it must confer some advantage over the risk-benefit profile of conventional approaches. The risks of traditional craniotomy approaches to the temporal lobe include neurological deficits, such as hemiparesis [16-18], cranial nerve deficits $[19,20]$, and visual field loss [6,21], and cognitive deficits, such as language and memory impairment $[22,23]$. Some of these complications can be attributed to the disruption of the lateral temporal cortex and white matter tracts. TEA has the potential to avoid this type of injury by providing a direct trajectory through the temporal pole and permitting earlier visualization of the surgical target (fig. 10). For example, the rate of visual field deficits secondary to the division of optic radiations is $55-76 \%$ after standard ATL $[6,21]$. The absence of visual field loss in our initial patients is therefore encouraging. This benefit alone may prove meaningful for patients' quality of life in light of the fact that up to one quarter of individuals with visual field cuts following ATL are in jeopardy of losing their driver's license even if they are seizure free [24]. Moreover, TEA affords the surgeon early visualization of the cranial nerves [7], which may facilitate the avoidance of iatrogenic injury. 
Traditional temporal approaches require a relatively large scalp incision, reflection or division of the temporalis muscle, and an open craniotomy. While well tolerated, these procedures can lead to the complications of cosmetic deformity, frontalis nerve palsy, and temporalis muscle wasting. TEA exchanges these risks for those associated with an eyelid or eyebrow incision. Although a facial incision may not be considered an appealing alternative, similar incisions are performed for plastic surgery 'face-lifts' to achieve cosmetic enhancements from a patient's baseline appearance. Thus, an excellent cosmetic result is possible using this type of incision.

Finally, endoscopic surgery is linked to a shorter hospital length of stay [25] and improved quality of life [26]. It is conceivable that TEA may follow this trend observed in other neuroendoscopic approaches.

\section{Potential Risks of TEA}

While TEA may provide certain benefits over traditional approaches, it also imposes new risks. The technique necessitates exposure of the lateral orbital wall and orbital retraction. While these maneuvers are standard practice in oculoplastics, they are not commonly performed by neurosurgeons and are associated with some risk to the globe. To evaluate the effect of orbital retraction in TEA, we carefully examined extraocular movements and visual acuity pre- and postoperatively. Both patients maintained their baseline ophthalmological exams immediately after surgery. This outcome is not surprising, given that the rate of ophthalmological change is less than $3 \%$ for medial transorbital approaches for extracranial indications [5, 8]. One patient experienced transient diplopia when watching television that spontaneously resolved. This symptom may have been related to lateral rectus muscle edema. No intervention was required. Larger patient cohorts will be required to compare the incidence of diplopia after TEA versus ATL, which is as high as $19 \%$ in some series $[19,20]$.

This novel strategy also requires careful consideration of dural and orbital reconstruction techniques to prevent CSF leakage. CSF leaks plagued early transnasal endoscopic surgeries, but the rates dropped substantially with the development of various surgical refinements. Patient B developed periorbital swelling following surgery, consistent with an orbital pseudomeningocele. She was offered an orbital reexploration with fat graft repair, but she opted for CSF diversion. Pseudomeningocele has not recurred after this subsequent intervention.

The significance of this pseudomeningocele remains to be determined. In the setting of pseudotumor cerebri, iatrogenic CSF leaks are purposely caused within the orbit by the creation of fenestrations within the optic nerve sheath. This procedure is well tolerated. In future cases, methods to reduce iatrogenic CSF leaks could be drawn from the transsphenoidal surgery literature. Although an easily accessible, minimally disfiguring pedicled flap, akin to the nasoseptal flap for anterior skull base approaches [27], is not available, the dural closure could be augmented with fat or fascia lata grafts. Pressure from the globe itself may also help buttress the closure. The rate and clinical impact of orbital pseudomeningoceles will need to be more carefully studied in larger series.

Finally, the operative time was longer in these patients than for traditional craniotomies. This finding was anticipated given the novel nature of the approach, and endoscopic operative times are known to decrease with experience [28]. A larger series may provide a better estimation of the duration of TEA once the nuances of this technique have been finalized.

\section{Conclusion}

We report the first clinical experience with the transorbital endoscopic approach to the medial temporal lobe through a lateral orbitotomy. Using this surgical alternative to temporal craniotomy, we successfully accessed the mesial temporal structures through a minimally 
Chen et al.: Lateral Transorbital Endoscopic Access to the Hippocampus, Amygdala and Entorhinal Cortex: Initial Clinical Experience

invasive incision. The procedure was accompanied by acceptable neurological, ophthalmological, and cosmetic outcomes. Surgical refinements are needed to decrease the operative time and minimize the rate of the CSF leak. A prospective study will permit this novel approach to be properly compared to traditional craniotomy.

\section{Disclosure Statement}

The authors do not have any disclosures or conflicts of interest to report.

\section{References}

1 Carrau RL, Jho HD, Ko Y: Transnasal-transsphenoidal endoscopic surgery of the pituitary gland. Laryngoscope 1996;106:914-918.

2 Jho HD, Carrau RL: Endoscopy assisted transsphenoidal surgery for pituitary adenoma. Technical note. Acta Neurochir (Wien) 1996;138:1416-1425.

3 Ali ZS, Lang SS, Kamat AR, Adappa ND, Palmer JN, Storm PB, Lee JY: Suprasellar pediatric craniopharyngioma resection via endonasal endoscopic approach. Childs Nerv Syst 2013;29:2065-2070.

4 Lang SS, Chen HI, Lee JY: Endoscopic microvascular decompression: a stepwise operative technique. Otorhinolaryngology 2012;74:293-298.

5 Moe KS, Bergeron CM, Ellenbogen RG: Transorbital neuroendoscopic surgery. Neurosurgery 2010;67:ons16ons28.

6 Wiebe S, Blume WT, Girvin JP, Eliasziw M: A randomized, controlled trial of surgery for temporal-lobe epilepsy. N Engl J Med 2001;345:311-318.

7 Chen HI, Bohman LE, Loevner L, Lucas TH: Transorbital endoscopic amygdalohippocampectomy: a feasibility investigation. J Neurosurg 2014;120:1428-1436.

8 Balakrishnan K, Moe KS: Applications and outcomes of orbital and transorbital endoscopic surgery. Otolaryngol Head Neck Surg 2011;144:815-820.

9 Bly RA, Ramakrishna R, Ferreira M, Moe KS: Lateral transorbital neuroendoscopic approach to the lateral cavernous sinus. J Neurol Surg B Skull Base 2014;75:11-17.

10 Bly RA, Su D, Lendvay TS, Friedman D, Hannaford B, Ferreira M, Moe KS: Multiportal robotic access to the anterior cranial fossa: a surgical and engineering feasibility study. Otolaryngol Head Neck Surg 2013;149: 940-946.

11 Ciporen JN, Moe KS, Ramanathan D, Lopez S, Ledesma E, Rostomily R, Sekhar LN: Multiportal endoscopic approaches to the central skull base: a cadaveric study. World Neurosurg 2010;73:705-712.

12 Silbergeld DL, Vollmer DG, Tantuwaya VS, Eichler ME: Endoscopic transventricular hippocampectomy. J Epilepsy 1995;8:68-73.

13 Willie JT, Laxpati NG, Drane DL, Gowda A, Appin C, Hao C, Brat DJ, Helmers SL, Saindane A, Nour SG, Gross RE: Real-time magnetic resonance-guided stereotactic laser amygdalohippocampotomy for mesial temporal lobe epilepsy. Neurosurgery 2014;74:569-584, discussion 584-565.

14 Barbaro NM, Quigg M, Broshek DK, Ward MM, Lamborn KR, Laxer KD, Larson DA, Dillon W, Verhey L, Garcia P, Steiner L, Heck C, Kondziolka D, Beach R, Olivero W, Witt TC, Salanova V, Goodman R: A multicenter, prospective pilot study of gamma knife radiosurgery for mesial temporal lobe epilepsy: seizure response, adverse events, and verbal memory. Ann Neurol 2009;65:167-175.

15 Regis J, Rey M, Bartolomei F, Vladyka V, Liscak R, Schrottner O, Pendl G: Gamma knife surgery in mesial temporal lobe epilepsy: a prospective multicenter study. Epilepsia 2004;45:504-515.

16 Wieser HG: Selective amygdalohippocampectomy has major advantages; in Miller JW, Silbergeld DL (eds): Epilepsy Surgery: Principles and Controversies. New York, Taylor and Francis, 2006, pp 465-478.

17 Rydenhag B, Silander HC: Complications of epilepsy surgery after 654 procedures in Sweden, September 1990-1995: a multicenter study based on the Swedish national epilepsy surgery register. Neurosurgery 2001; 49:51-56, discussion 56-57.

18 Behrens E, Schramm J, Zentner J, Konig R: Surgical and neurological complications in a series of 708 epilepsy surgery procedures. Neurosurgery 1997;41:1-9, discussion 9-10.

19 Cohen-Gadol AA, Leavitt JA, Lynch JJ, Marsh WR, Cascino GD: Prospective analysis of diplopia after anterior temporal lobectomy for mesial temporal lobe sclerosis. J Neurosurg 2003;99:496-499.

20 Sindou M, Guenot M, Isnard J, Ryvlin P, Fischer C, Mauguiere F: Temporo-mesial epilepsy surgery: Outcome and complications in 100 consecutive adult patients. Acta Neurochir (Wien) 2006;148:39-45.

21 Egan RA, Shults WT, So N, Burchiel K, Kellogg JX, Salinsky M: Visual field deficits in conventional anterior temporal lobectomy versus amygdalohippocampectomy. Neurology 2000;55:1818-1822.

22 Acar G, Acar F, Miller J, Spencer DC, Burchiel KJ: Seizure outcome following transcortical selective amygdalohippocampectomy in mesial temporal lobe epilepsy. Stereotact Funct Neurosurg 2008;86:314-319. 
Chen et al.: Lateral Transorbital Endoscopic Access to the Hippocampus, Amygdala and Entorhinal Cortex: Initial Clinical Experience

23 Jensen I: Temporal lobe surgery around the world. Results, complications, and mortality. Acta Neurol Scand 1975;52:354-373.

24 Manji H: Epilepsy surgery, visual fields, and driving: a study of the visual field criteria for driving in patients after temporal lobe epilepsy surgery with a comparison of Goldmann and Esterman perimetry. Am J Ophthalmol 2000;129:704.

25 Cappabianca P, Alfieri A, Colao A, Ferone D, Lombardi G, de Divitiis E: Endoscopic endonasal transsphenoidal approach: an additional reason in support of surgery in the management of pituitary lesions. Skull Base Surg 1999;9:109-117.

26 McCoul ED, Anand VK, Schwartz TH: Improvements in site-specific quality of life 6 months after endoscopic anterior skull base surgery: a prospective study. J Neurosurg 2012;117:498-506.

27 Hadad G, Bassagasteguy L, Carrau RL, Mataza JC, Kassam A, Snyderman CH, Mintz A: A novel reconstructive technique after endoscopic expanded endonasal approaches: vascular pedicle nasoseptal flap. Laryngoscope 2006;116:1882-1886.

28 O'Malley BW Jr, Grady MS, Gabel BC, Cohen MA, Heuer GG, Pisapia J, Bohman LE, Leibowitz JM: Comparison of endoscopic and microscopic removal of pituitary adenomas: single-surgeon experience and the learning curve. Neurosurg Focus 2008;25:E10. 